\title{
Mobile App-based contact Tracing During COVID-19-Simple and Without Recall Bias
}

\author{
Shivangi Saha ${ }^{1} \cdot$ Praveen Prakash $^{1} \cdot$ Seema Singhal ${ }^{2} \cdot$ Shashank Chauhan $^{1,3} \cdot$ \\ Maneesh Singhal ${ }^{1}$. Shruthi Chandrashekar ${ }^{1}$
}

Received: 8 September 2020 / Accepted: 7 January 2021 / Published online: 16 January 2020

(C) Association of Surgeons of India 2021

In the context of the COVID-19 pandemic, contact tracing is a key mitigation strategy. It is of paramount importance amongst health care personnel as they are a high-risk group $[1,2]$.

We work in a busy surgical department of a tertiary care centre in India. As part of the COVID-19 infection control strategy, contact tracing amongst all healthcare workers was mandated at our institute and each department has delegated the task to conduct contact tracing for any member who is symptomatic or positive for COVID19. Initially, we adopted the traditional Interview based contact tracing (IBCT) which, we realised, had several disadvantages. IBCT took a significant amount of time, had no active motivation for healthcare workers to keep track of their contacts and other specific details, and in the event of an actual positive case being identified, a recall could be affected by psychological factors like anxiety and stress [3, 4]. These factors could make the process of contact tracing less efficacious and hence, negatively impact the safety of our health care personnel.

To address these concerns, we decided to implement an online data collection platform with a predefined questionnaire which would allow for the members to make entries at any time. This would improve uniformity and completeness in

Shashank Chauhan

drshashankchauhan@gmail.com

Shivangi Saha

shivangisaha@gmail.com

Praveen Prakash

prav94@gmail.com

Seema Singhal

drseemasinghal@gmail.com

Maneesh Singhal

drmaneesh@gmail.com data collection. A dedicated native smartphone application would make the process more intuitive and convenient since all members generally have a smartphone with them during work hours.

To avoid the substantial costs and resources needed for developing, configuring, deploying and maintaining the software infrastructure ourselves, we opted to use Epicollect5, an online data collection platform which met our requirements and was completely free to use.

A cross-sectional study was performed from April to June 2020 (Fig. 1). Thirty-two members of the department (23 residents, 4 physiotherapists and 5 faculty members) participated in the study. Ten rounds of mock drills were conducted using IBCT (Phase 1). Subsequently, online self-reported contact tracing (OSRCT) using the Epicollect5 mobile data collection platform was implemented, where participants self-reported all significant contacts on a smartphone application within $24 \mathrm{~h}$. Furthermore, ten rounds of mock drills using this online database were performed (Phase 2). Time taken for both the methods and data relating to a number of contacts were recorded and compared (Table 1).

OSRCT took significantly less time for reporting compared to the IBCT (74.5 SD 12.8 vs $143.5 \mathrm{SD} 28 \mathrm{~min}, p<0.001$ ). There was no significant difference in the number of contacts

Shruthi Chandrashekar shruthic_24@yahoo.com

Department of Plastic, Reconstructive and Burns Surgery, All India Institute of Medical Sciences, New Delhi, India

2 Department of Obstetrics and Gynaecology, All India Institute of Medical Sciences, New Delhi, India

Department of Plastic, Reconstructive \& Burns Surgery, All India Institute of Medical Sciences, Room no. 207, Faculty offices, 2nd Floor, Burns and Plastic Surgery Block (AIIMS), JPNATC Campus, Raj Nagar, New Delhi 110029, India 
Fig. 1 Timeline of events, process flowcharts and data collected from various phases of the study

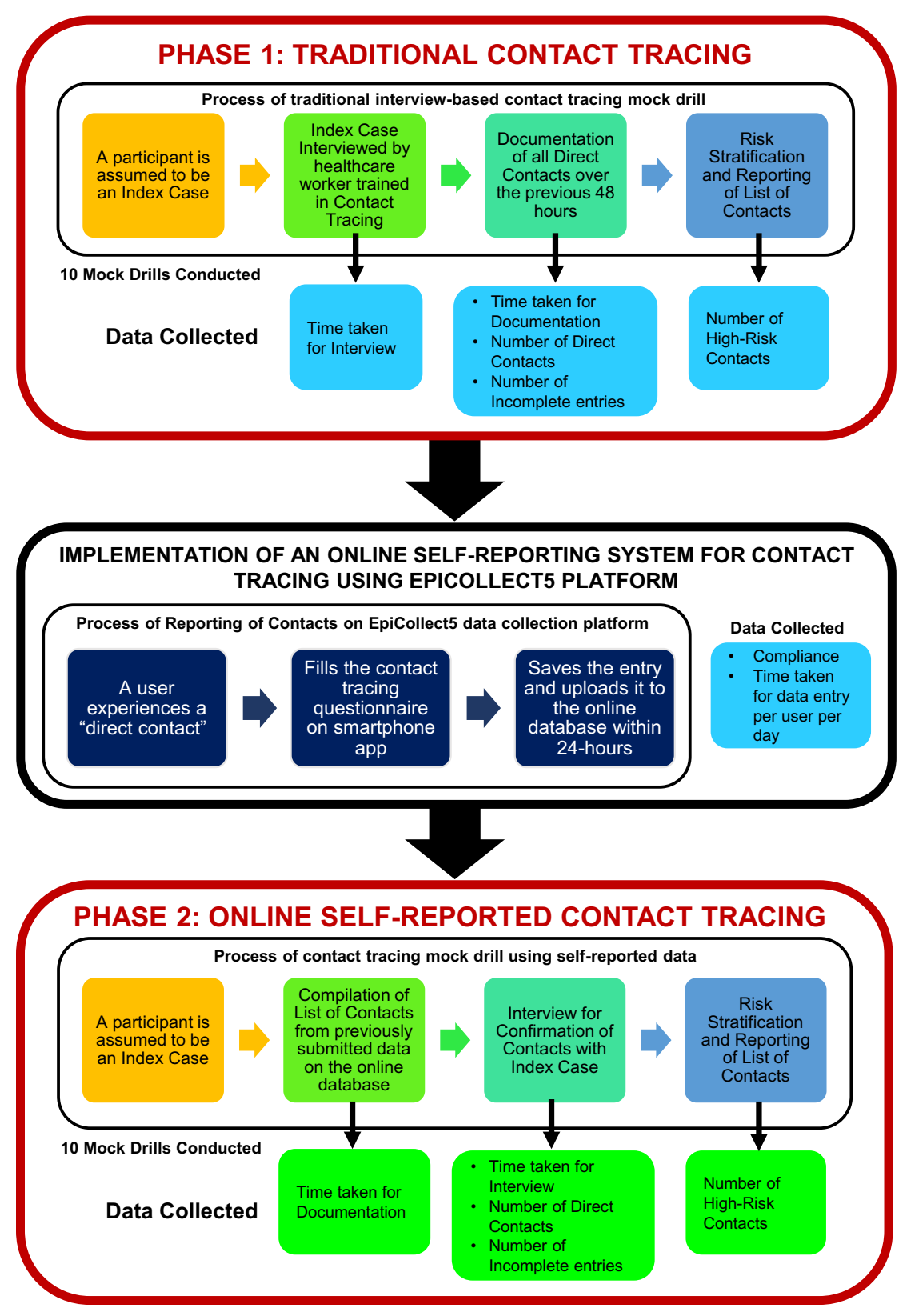

Table 1 Data from mock contact tracing drills conducted using interview-based contact tracing and online self-reported contact tracing

\begin{tabular}{|c|c|c|c|c|c|}
\hline \multirow[t]{2}{*}{ Parameter } & \multicolumn{2}{|c|}{$\begin{array}{l}\text { Interview based contact tracing mock drills }(n \\
=10)\end{array}$} & \multicolumn{2}{|c|}{$\begin{array}{l}\text { Online self-reported contact tracing mock drills } \\
(n=10)\end{array}$} & \multirow[t]{2}{*}{$p$ values* } \\
\hline & Mean & $\mathrm{SD}$ & Mean & $\mathrm{SD}$ & \\
\hline Time taken for interview (min) & 60.5 & 12.7 & 34.5 & 7.57 & $<0.001$ \\
\hline Time taken for documentation (min) & 83 & 18.8 & 40 & 7.4 & $<0.001$ \\
\hline Time to final report (min) & 143.5 & 28.0 & 74.5 & 12.8 & $<0.001$ \\
\hline Total time taken for contact tracing (min) & 143.5 & 28.0 & 91.9 & 16.3 & $<0.001$ \\
\hline Total number of direct contacts & 31.2 & 7.2 & 29.6 & 6.8 & 0.63 \\
\hline Number of high-risk contacts & 3.8 & 1.3 & 3.2 & 0.8 & 0.25 \\
\hline Number of incomplete contacts & 5.5 & 1.5 & 4.5 & 1.7 & 0.17 \\
\hline
\end{tabular}

* $p$ value calculated using the unpaired $t$ test 
recorded. The compliance to self-reporting reached $100 \%$ by the fourth-day post-implementation.

The system had several other advantages. Users could report their contacts at their convenience without giving a protracted and potentially stressful interview. Unlike recall from memory, the quality of the data does not deteriorate over time as it is stored digitally, which is especially advantageous when the recall period crosses $48 \mathrm{~h}$ [5]. The system can also encourage positive behavioural changes whereby users are vigilant to avoid non-essential contacts and maintain social distancing.

\section{Compliance with Ethical Standards}

Conflict of Interest The authors declare that they have no conflict of interest.

Ethical Approval Approved by the Institute Ethics Committee (Ref. No IECPG-262/24.06.2020).

\section{References}

1. den Bergh MFQK, Buiting AGM, Pas SD, Bentvelsen RG, van den Bijllaardt W, van Oudheusden AJG et al (2020) Prevalence and clinical presentation of health care workers with symptoms of coronavirus disease 2019 in 2 Dutch hospitals during an early phase of the pandemic. JAMA Netw Open 3:e209673-e209673. https://doi. org/10.1001/jamanetworkopen.2020.9673

2. Zabarsky TF, Bhullar D, Silva SY, Mana TS, Ertle MT, Navas ME, Donskey CJ (2020) What are the sources of exposure in healthcare personnel with coronavirus disease 2019 infection? Am J Infect Control.

3. Saurabh S, Prateek S (2017) Role of contact tracing in containing the 2014 Ebola outbreak: a review. Afr Health Sci 17:225-236. https:// doi.org/10.4314/ahs.v17i1.28

4. Mosser AE, Evans JR (2019) Increasing the number of contacts generated during contact tracing interviews. Mem Hove Engl 27: 495-506. https://doi.org/10.1080/09658211.2018.1529247

5. Murre JMJ, Dros J (2015) Replication and analysis of Ebbinghaus' forgetting curve. PLoS One 10:e120644. https://doi.org/10.1371/ journal.pone.0120644

Publisher's Note Springer Nature remains neutral with regard to jurisdictional claims in published maps and institutional affiliations. 